\title{
The Effects of Hearing Aid Digital Noise Reduction and Directionality on Acceptable Noise Level
}

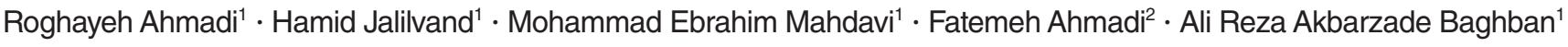 \\ ${ }^{1}$ Department of Audiology, School of Rehabilitation, Shahid Beheshti University of Medical Sciences, Tehran; \\ ${ }^{2}$ School of Economic, Allameh Tabataba'i University, Tehran, Iran
}

Objectives. Two main digital signal processing technologies inside the modern hearing aid to provide the best conditions for hearing aid users are directionality (DIR) and digital noise reduction (DNR) algorithms. There are various possible settings for these algorithms. The present study evaluates the effects of various DIR and DNR conditions (both separately and in combination) on listening comfort among hearing aid users.

Methods. In 18 participants who received hearing aid fitting services from the Rehabilitation School of Shahid Beheshti University of Medical Sciences regularly, we applied acceptable noise level (ANL) as our subjective measure of listening comfort. We evaluated both of these under six different hearing aid conditions: omnidirectional-baseline, omnidirectional-broadband DNR, omnidirectional-multichannel DNR, directional, directional-broadband DNR, and directional-multichannel DNR.

Results. The ANL results ranged from $-3 \mathrm{~dB}$ to $14 \mathrm{~dB}$ in all conditions. The results show, among all conditions, both the omnidirectional-baseline condition and the omnidirectional-broadband DNR condition are the worst conditions for listening in noise. The DIR always reduces the amount of noise that patients received during testing. The DNR algorithm does not improve listening in noise significantly when compared with the DIR algorithms. Although both DNR and DIR algorithms yielded a lower ANL, the DIR algorithm was more effective than the DNR.

Conclusion. The DIR and DNR technologies provide listening comfort in the presence of noise. Thus, user benefit depends on how the digital signal processing settings inside the hearing aid are adjusted.

Keywords. Directional Hearing Aid; Hearing Aids; Acceptable Noise Level; Hearing Loss; Digital Noise Reduction

\section{INTRODUCTION}

The role of the auditory system is detection, discrimination, and identification of various sounds and noises. The main function of this system for human beings is speech perception, known as speech intelligibility, especially in difficult auditory conditions, such as when there is noise. One of the main complaints of patients with sensory neural hearing loss is the problem of speech

- Received January 14, 2018

Revised March 10, 2018

Accepted April 17, 2018

- Corresponding author: Hamid Jalilvand

Department of Audiology, School of Rehabilitation, Shahid Beheshti

University of Medical Sciences, Damavand St.,Tehran, Iran

Tel: +98-21-7756-1721, Fax: +98-21-7759-1807

E-mail: hamidjalilvand@sbmu.ac.ir intelligibility in babble noise, which cannot be improved while using a hearing aid [1,2]. For listening comfort and speech intelligibility in noise, there are two main digital signal processing (DSP) technologies, the digital noise reduction (DNR) and directionality (DIR), which are used when fitting current modern digital hearing aids. The DNR approach, which separates sounds based on their frequency and reduces the hearing aid gain into frequency bands with lower signal-to-noise ratios (SNRs), provides listening comfort in noise. It has been shown DNR reduces adverse effects of noise on memory for speech, helps the hearing aid user in noisy conditions, and prevents cognitive load during auditory processing $[3,4]$. On the other hand, DIR, which separates sounds based on a spatial approach, focuses on a specific direction (most commonly, in front), receives a target signal (speech) the hearing aid user probably prefers to hear (i.e., a tar-

Copyright ( 2018 by Korean Society of Otorhinolaryngology-Head and Neck Surgery.

This is an open-access article distributed under the terms of the Creative Commons Attribution Non-Commercial License (http://creativecommons.org/licenses/by-nc/4.0)

which permits unrestricted non-commercial use, distribution, and reproduction in any medium, provided the original work is properly cited. 
get speech in a group), and improves the SNR of the listening environment [5]. These two algorithms inside a digital hearing aid work together to provide optimal amplification coupled with the highest possible speech intelligibility in noise.

Although it has been demonstrated DNR algorithms provide an improvement in subjective measurements [6,7], few studies show the objective improvement provided by DNR [8]. Typically, in some specific hearing aids, the DNR algorithm can be fitted as two main types: broadband DNR and multichannel DNR. The former algorithm involves gain reduction by a default amount through a whole range of hearing aid gain frequency responses when the hearing aid detects noise. Under this condition, the gain is decreased proportionally in the overall frequency range (Supplementary Table 1, Supplementary Figs. 1-5). The latter algorithm involves a specific amount of gain reduction that is both determined and limited by the audiologist during the hearing aid fitting. In a multichannel DNR, by applying various settings, there could be different DNR conditions. In clinical practice, there are no generally accepted rationale for selecting comparable DNR algorithms and settings when fitting modern hearing aids [9]. Practically, they are adjusted in terms of the audiologist's experience and are based on clinical trial protocols or specific procedures recommended by hearing aid companies. Also, there has been no study to both compare broadband DNR with multichannel DNR and the various conditions of multichannel DNR.

In this study, we used the acceptable noise level (ANL) as our measurement of subjective comfort [10]. We compared both the effectiveness of DIR and two different DNR settings (broadband vs. multichannel DNR) separately. In addition, we compared the efficacy of both algorithms (DNR and DIR) by measuring the ANL. The ANL was chosen because it has been shown the ANL can prove the effectiveness of noise reduction is provided by DNR algorithms $[8,11]$. Our main purpose is to evaluate the effectiveness of DIR and DNR technologies (both alone and in combination) on patient performance in noise.

\section{H I G G H L I I G H T S}

- Among all conditions, both the omnidirectional-baseline condition and the omnidirectional-broadband digital noise reduction (DNR) condition are the worst conditions for listening in noise.

- The directionality (DIR) always reduces the amount of noise that patients received during testing.

- The DNR algorithm does not improve listening in noise significantly when compared with the DIR algorithms.

- Although it only reduces the amount of noise compared with the omnidirectional condition, the DNR must be set to the broadband condition rather than the multichannel condition.

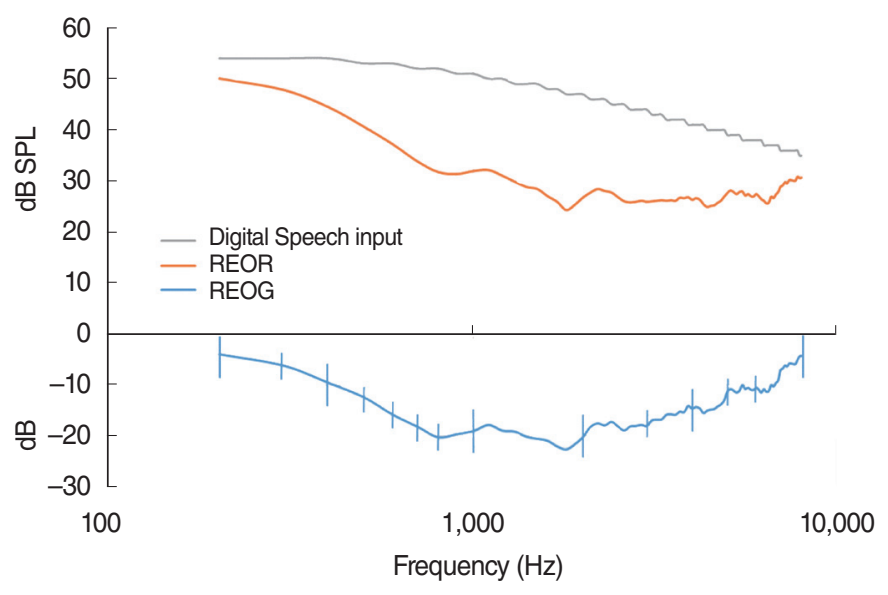

Fig. 1. Real ear occluded gain (REOG) in $d B$, across frequencies $(200-8,000 \mathrm{~Hz})$ in opposite ear that is occluded by a tight ear impression. The original signal input (Digital Speech stimulus, average root mean of square of $65 \mathrm{~dB}$ sound pressure level [SPL]) and real ear occluded response (REOR) are shown in $\mathrm{dB} S \mathrm{SPL}$. In order to obtain the REOG, the input signal level is subtracted from the REOR across frequencies. Values are presented as mean \pm standard deviation.

\section{MATERIALS AND METHODS}

\section{Participants}

Eighteen patients (eight males and 10 females) with moderate sloping sensorineural hearing loss, who received hearing aid fitting services from the Rehabilitation School of Shahid Beheshti University of Medical Sciences (SBMU) regularly, participated in the study. All participants, who were randomly and blindly selected by research secretary of Audiology Department, were fitted monaurally (10 right ears, eight left ears). During all tests, the opposite ear was occluded by a tight ear impression. On average, the hearing thresholds were increased in range of 5-20, $20-25$, and $5-15 \mathrm{~dB}$ for low, mid, and high frequency range that were consistent with mean real ear occluded gain measurement, which was obtained from all occluded ears (Fig. 1). All participants were experienced users of hearing aids (at least 2 years of experience using hearing aids). To control the probable effect of audiogram configuration on the ANL and meet our aim for both homogeneity of audiogram pattern and degree of hearing loss among subjects, we determined our inclusion criterion to be mild to moderate sloping hearing loss, not worse than $75 \mathrm{~dB} \mathrm{HL}$ in $4,000 \mathrm{~Hz}$ and $8,000 \mathrm{~Hz}$ [12]. The average audiogram for each ear is shown in Fig. 2, which indicates similar average thresholds between test ears and test groups. Also, the average audiogram for each group is shown in Fig. 2. All participants were native Persian speakers, and their average age was $64.56 \pm 19$ years. Before participating in the study, the memory capacity of each subject was evaluated by the Mini-Mental State Examination (MMSE). The criterion for participating in the present study was a cutoff score of 23 or higher on the MMSE [13]. After passing 


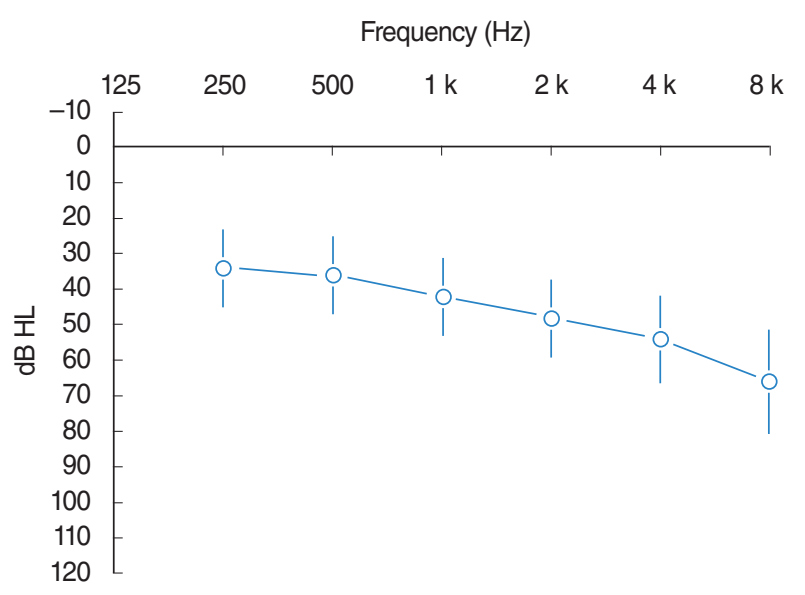

Fig. 2. Hearing thresholds for test ears. Values are presented as mean \pm standard deviation.

the screening MMSE, both the aim of the study and its procedures were explained to all subjects and signed informed consent was obtained from all subjects.

All test procedures were conducted in two sessions. The first session was to explain the study purpose, sign the consent form, perform audiometry and immittance tests, and take the ear impression to provide an individual earmold for each participant. The second session was to fit and adjust the hearing aid with his or her earmold, perform real ear measurement, and conduct the ANL test to counterbalance and assign subjects randomly to the various DIR and DNR conditions. Each session was separated by 2 weeks and required 1.5-2.5 hours to complete so that resting time (15-30 minutes) was considered during testing, if necessary. The ethical committee of the SBMU approved all the used procedures, methods, and tests (IR.SBMU.RETECH.REC.1396. 183).

\section{Hearing aid fitting}

To provide the similar technical benefits of DIR and DNR algorithms, we fitted the same BTE hearing aid in all participants. In the present study, we used the Siemens Motion 301 P BTE (Siemens Hearing Instruments Inc., Erlangen, Germany), which employs eight-channel wide dynamic range compression with multichannel DIR. As described comprehensively by Mueller et al. [11], the DNR by Siemens Co. employs two DNR algorithms: modulation based DNR and adaptive fast-acting DNR. Modulation based DNR, which is a relatively slow acting, is designed to decrease the gain inside a channel when the presence of steadystate noise is detected. The detection of steady-state noise is based on its modulation, and the amount of gain reduction can be set during the fitting session. The adaptive fast-acting DNR, which is similar to Wiener filter technology, tracks the signal envelope in each channel. Next, it calculates the SNR and the filter coefficient for that channel. Inside each channel, the calculated filter coefficient is applied directly to the signal. This type of
Table 1. Six different conditions of DNR and DIR settings tested on participants

\begin{tabular}{|c|c|c|c|}
\hline $\begin{array}{l}\text { Condition } \\
\text { no. }\end{array}$ & Name of condition & DNR settings & DIR settings \\
\hline 1 & $\begin{array}{l}\text { Omnidirectional-baseline } \\
\text { condition }\end{array}$ & Off & $\begin{array}{l}\text { Omnidirectional } \\
\text { microphone }\end{array}$ \\
\hline 2 & $\begin{array}{l}\text { Omnidirectional-broad- } \\
\text { band DNR condition }\end{array}$ & On-broadband & $\begin{array}{l}\text { Omnidirectional } \\
\text { microphone }\end{array}$ \\
\hline 3 & $\begin{array}{l}\text { Omnidirectional-multi- } \\
\text { channel DNR condition }\end{array}$ & On-multichannel & $\begin{array}{l}\text { Omnidirectional } \\
\text { microphone }\end{array}$ \\
\hline 4 & Directional condition & Off & $\begin{array}{l}\text { Directional } \\
\text { microphone }\end{array}$ \\
\hline 5 & $\begin{array}{l}\text { Directional-broadband } \\
\text { DNR condition }\end{array}$ & On-broadband & $\begin{array}{l}\text { Directional } \\
\text { microphone }\end{array}$ \\
\hline 6 & $\begin{array}{l}\text { Directional-multichannel } \\
\text { DNR condition }\end{array}$ & On-multichannel & $\begin{array}{l}\text { Directional } \\
\text { microphone }\end{array}$ \\
\hline
\end{tabular}

DNR, digital noise reduction; DIR, directionality.

DNR is always active regardless of the presence of modulations in the input signal.

In the early phase of the study, the ear impression was provided for each participant to make an unvented hard canal earmold. Regarding the inevitable effects of the open fitting on SNR improvement provided by DIR as well as on DNR function [14-16], a closed fitting was completed for all subjects, regardless of near normal hearing in the low frequency range (10 participants had hearing thresholds less than $35 \mathrm{~dB} \mathrm{HL}$ at $250 \mathrm{~Hz}$ and $500 \mathrm{~Hz}$ ). The fitting software SiFit (v6.11; Siemens Audiology Solutions, Erlangen, Germany) and rationale National Acoustic Laboratories-Nonlinear 2 (NAL-NL2) was used to program the hearing aid. Real ear measurement was taken using an FP35 instrument (Frye Electronics, San Jose, CA, USA), and the real ear aided response was measured by the broadband stimulus, Digital Speech, with levels of 50,65, and $80 \mathrm{~dB}$ sound pressure level (SPL) to represent the soft, medium, and high inputs, respectively. Hearing aid amplification had to be set regarding \pm 3 $\mathrm{dB}$ range of NAL-NL2 targets for all inputs. Amplitude compression settings were left on the Siemens default base. In addition, to control the probable effects of maximum pressure output (MPO) settings on the results [17], short, pure tones with a level of $90 \mathrm{~dB}$ SPL were used for both measuring and adjusting the hearing aid MPO. During all testing conditions, the other DSP algorithms such as SoundSmoothing, eWindScreen, and Feedback Stopper were inactivated. For each subject, there were a total of six different conditions of DNR and DIR settings for testing (Table 1).

For DNR settings, there are three different conditions: (1) DNR is off (there is no gain reduction when the hearing aid identifies the noise); (2) DNR is on, and the broadband setting is chosen (there is an 8-15 $\mathrm{dB}$ gain reduction in all frequency ranges of the hearing aid after identifying the noise); and (3) DNR is on, and the multichannel setting is chosen, and the Max, Med, and Off settings are selected as different strengths of the 
DNR algorithm for gain reduction in frequencies bands of 100 $500,500-1,400$, and $1,400-8,000 \mathrm{~Hz}$, respectively. There is a maximum gain reduction for frequencies lower than $500 \mathrm{~Hz}$, a minimum gain reduction for $500-1,400 \mathrm{~Hz}$, and no gain reduction for frequencies higher than $1,400 \mathrm{~Hz}$.

For DIR settings, there are only two different conditions: (1) omnidirectional microphone, and (2) directional microphone (uses dual microphones and has multichannel adaptive DIR).

The above different settings of DNR and DIR, which totaled six conditions (Table 1), were selected by SiFit software randomly for the ANL test. In addition, we measured the DNR reaction of the mentioned hearing aid by the 2-cc coupler measurement, which was performed using the Digital Speech and the Composite Noise stimuli to represent speech and noise, respectively (Supplementary Table 1, Supplementary Figs. 1-5).

\section{Test materials}

Acceptable noise level

Nabelek et al. [10] introduced the ANL as a test for quantifying the patient's tolerance of background noise while listening to a running speech. In this study, the Persian version of the ANL was used and was provided and validated by the Audiology Department of Tehran University of Medical Science [18]. This version has a running story with a female speaker and background noise (a 12-speaker babble noise). In the anechoic booth, both speech and noise stimuli were presented by a CD player and a calibrated audiometer (ANSI S3.6-2004, Specification for audiometers, Acoustical Society of America, Melville, NY, USA) via speakers located $1 \mathrm{~m}$ away from the subject in his or her front and rear, respectively.

Before testing, every participant was given both verbal and written instructions describing the test procedure and his or her task. For determining the ANL, two typical main measurements have to be evaluated: most comfortable level (MCL) and Background Noise Level (BNL). First, for the MCL measurement, running female speech (in quiet) is presented in $30 \mathrm{~dB} \mathrm{HL}$ via the front speaker. The speech level is increased or decreased in steps of $5 \mathrm{~dB}$, by her or his thumb signal (up or down for increasing and decreasing the level, respectively). The patient was asked to signal the level adjustment until her/his MCL was reached. To find the exact MCL, the levels of speech presented were significantly lower and higher than her/his MCL. Next, the subject was asked again to compare her/his MCL with these levels and readjust the MCL. After MCL readjustment, the speech level was increased or decreased in $2 \mathrm{~dB}$ steps. The final preferred level was recorded as her/his MCL. The MCL measurement was performed twice, and the average was considered as the final MCL.

The BNL measurement is the second part of the ANL test. For its measurement, while presenting running speech at the fixed level (the measured MCL), babble noise is presented at 30 $\mathrm{dB}$ HL via the rear speaker. Again, the noise level is increased or decreased, in steps of $5 \mathrm{~dB}$, by her/his thumb signal. The patient was instructed to determine the noise level they could no longer tolerate and be able to follow the running speech. During the final stage of BNL measurement, the noise level was increased or decreased in $2 \mathrm{~dB}$ steps. In addition, after determining the BNL, the speech in noise continued to play for an additional $10 \mathrm{sec}-$ onds to provide sufficient time for both activation and establishment of DSP algorithms inside the hearing aid and the participant's decision on her/his final BNL. The BNL was measured three times, and the average was considered as the BNL. Finally, for the ANL calculation, the BNL was subtracted from MCL (ANL=MCL-BNL). The lower ANL suggests the participants could tolerate the higher level of noise. The ANL was conducted for the six different aided conditions (Table 1).

\section{Statistical methods}

To analyze the study results, IBM SPSS ver. 24.0 (IBM Corp., Armonk, NY, USA) was used. The statistical descriptive parameters such as the mean and standard deviation of the ANL were considered. To determine the statistically significant differences among the various conditions of the ANL, at the 0.05 level, a one-way repeated-measurement analysis of variance (ANOVA) test was performed. In addition, while comparing the various conditions, the Bonferroni test as a post hoc test was used. In this analysis, the within subject factor consisted of six various conditions.

\section{RESULTS}

\section{Acceptable noise level}

The ANL results ranged from $-3 \mathrm{~dB}$ to $14 \mathrm{~dB}$ in all six conditions. The ANL means were $6.00,4.33,5.00,2.67,2.28$, and $1.56 \mathrm{~dB}$ for omnidirectional-baseline, omnidirectional-broadband DNR, omnidirectional-multichannel DNR, directional, directional-broadband DNR, and directional-multichannel DNR conditions, respectively. Scatterplots comparing the individual ANL results between various hearing aid conditions are shown in Fig. 3. To facilitate comparison, based on an approach by Peeters et al. [8], the omnidirectional-baseline condition, which is the baseline condition and all participants who had poor performance, was plotted as the horizontal axis (X-axis) while the other five conditions, which may help improve performance in all subjects, was plotted as the vertical axis (Y-axis). Consequently, if there is no difference between the omnidirectionalbaseline condition and each DIR and DNR condition, the data points are found on or near the diagonal line. On the other hand, if the performance of subjects would be better by using each DIR and DNR condition, the data points would fall below the diagonal, which indicates the specific hearing aid condition defined by the Y-axis helps improve performance than the condition defined by the $\mathrm{X}$-axis. 

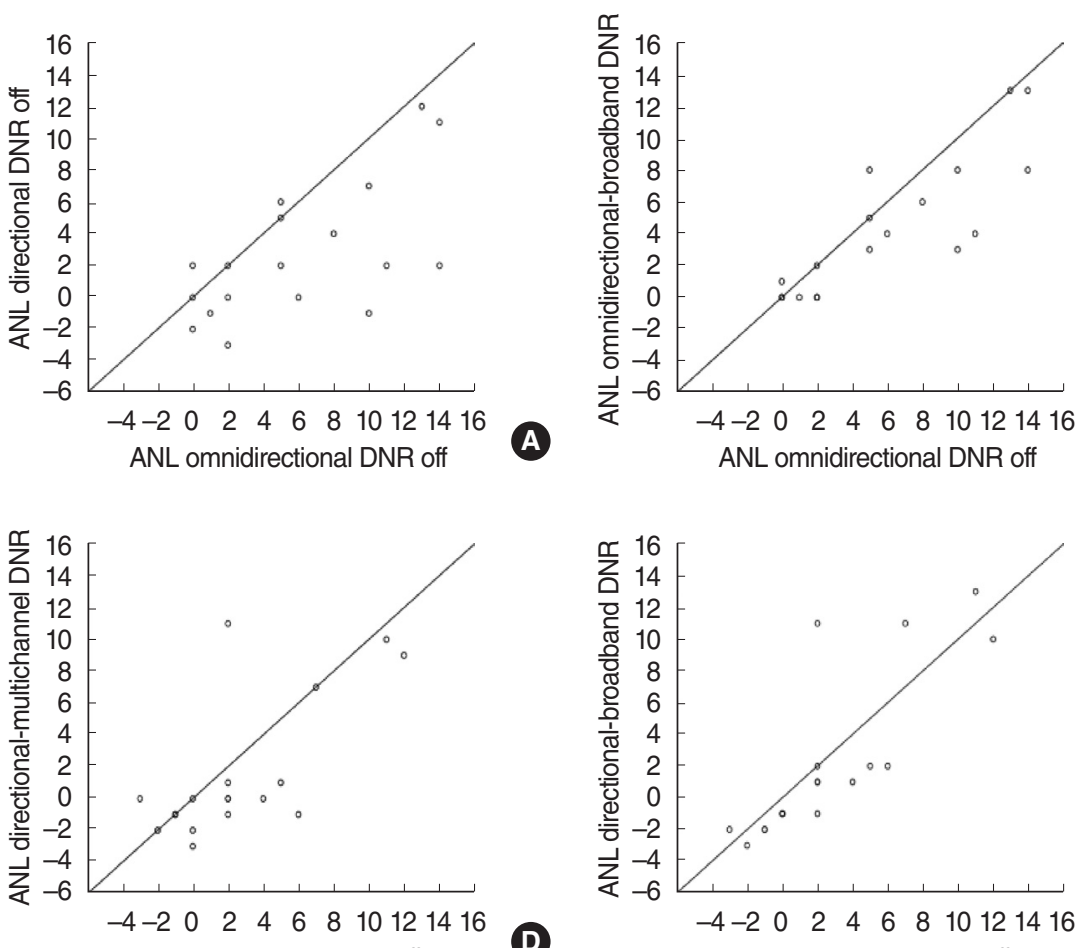

ANL directional DNR off

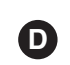

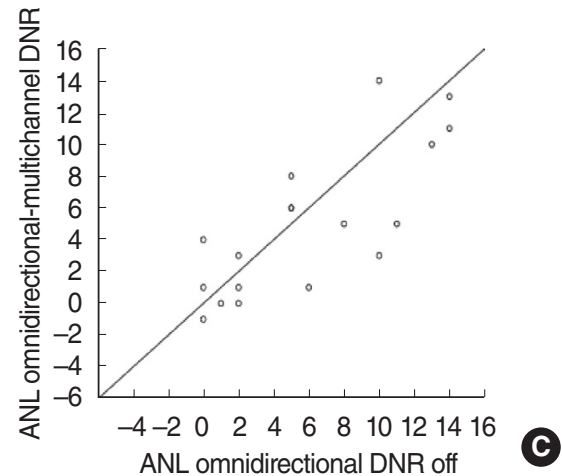

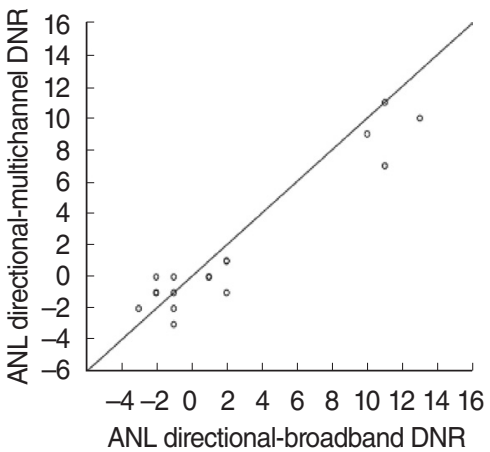

Fig. 3. Scatterplots showing the acceptable noise level (ANL) in $\mathrm{dB}$ for individual participants between two conditions. The comparisons are (A) omnidirectional-baseline and directional conditions, (B) omnidirectional-baseline and omnidirectional-broadband digital noise reduction (DNR) conditions, (C) omnidirectional-baseline and omnidirectional-multichannel DNR conditions, (D) directional and directional-multichannel DNR conditions, (E) directional and directional-broadband DNR conditions, and (F) directional-broadband DNR and directional-multichannel DNR conditions. Points above the diagonal line reflect better performance with hearing aid condition represented by the horizontal axis. Points below the diagonal line show better performance with the hearing aid condition represented by the vertical axis.

One-way repeated measures ANOVA of the ANL of the six hearing aid conditions was significant $\left(\mathrm{F}_{(1,16)}=47.901, P<0.001\right)$. Post hoc analysis with adjustment for multiple comparisons using Bonferroni corrections showed there were statistically significant differences among the omnidirectional-baseline condition and omnidirectional-broadband DNR condition $(P<0.005)$, the directional condition $(P<0.005)$, the directional-broadband condition $(P<0.003)$, and the directional-multichannel DNR condition $(P<0.001)$, except when the omnidirectional-baseline condition was compared with the omnidirectional-multichannel DNR condition (Fig. 4).

Also, there were statistically significant differences among the omnidirectional-broadband DNR condition and the directional condition $(P<0.040)$, the directional-broadband DNR condition $(P<0.030)$, and the directional-multichannel DNR condition $(P<0.005)$ (Fig. 4). Similarly, there were statistically significant differences among the omnidirectional-multichannel DNR condition and the directional condition $(P<0.005)$, the directionalbroadband DNR condition $(P<0.001)$, and the directional-multichannel DNR condition $(P<0.002)$ (Fig. 4). These results show first, among all conditions, both the omnidirectional-baseline condition and the omnidirectional-broadband DNR condition are the worst conditions for listening in noise. Second, the DIR always reduces the amount of noise that patients received during testing. Third, the DNR algorithm does not improve listening in noise significantly when compared with the DIR algorithms. Although it only reduces the amount of noise compared with the omnidirectional condition, the DNR must be set to the broadband condition rather than the multichannel condition.

\section{ANL benefit}

The ANL benefit, which is the difference between a specific condition and omnidirectional-baseline condition, was calculated for all participants under the five DNR and DIR conditions (Fig. 5). The maximum and minimum benefits belonged to the directional-multichannel DNR condition and the omnidirectionalmultichannel condition, respectively. Generally, the total average ANL benefit decreased from the directional-multichannel DNR condition $(4.44 \mathrm{~dB})$, to the directional-broadband DNR condition $(3.72 \mathrm{~dB})$, the directional condition $(3.33 \mathrm{~dB})$, the broadband DNR condition $(1.66 \mathrm{~dB})$, and the multichannel DNR condition $(1.00 \mathrm{~dB})$. As indicated in Fig. 5, the DIR algorithm provides the maximum ANL benefit to all the other conditions whereas the multichannel DNR algorithm provides additional 


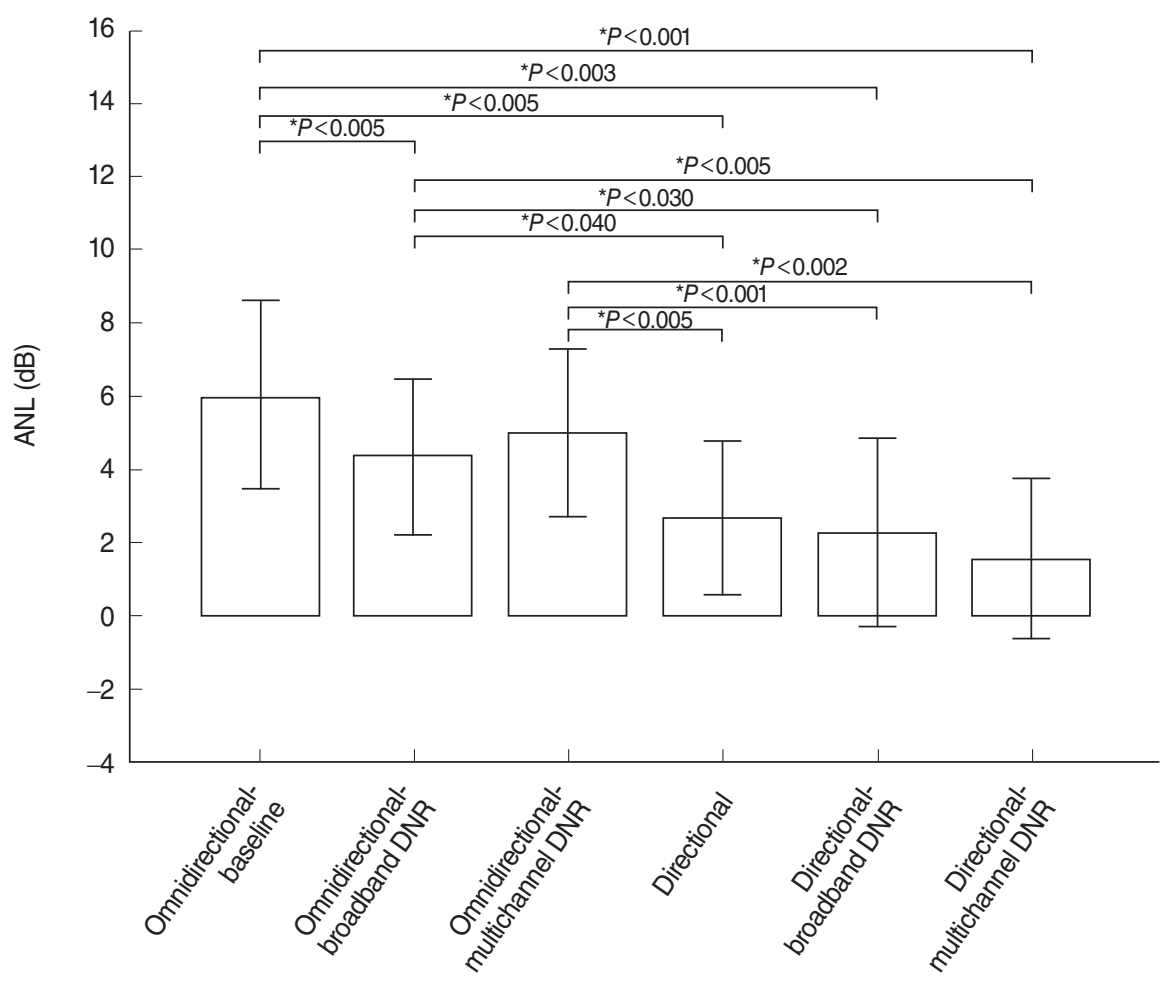

Fig. 4. The mean acceptable noise level (ANL) for the six digital noise reduction (DNR) and directionality conditions. There are statistically significant differences among the omnidirectional-baseline condition and omnidirectional-broadband DNR condition, directional condition, directional-broadband DNR condition, and directional-multichannel DNR condition. In addition, there are statistically significant differences among the omnidirectional-broadband DNR condition and directional condition, directional-broadband DNR condition, and directional-multichannel DNR condition. Also, there are statistically significant differences among the omnidirectional-multichannel DNR condition and directional condition, directional-broadband DNR condition, and directional-multichannel DNR condition. The asterisks show the statistical significant difference for each comparison.

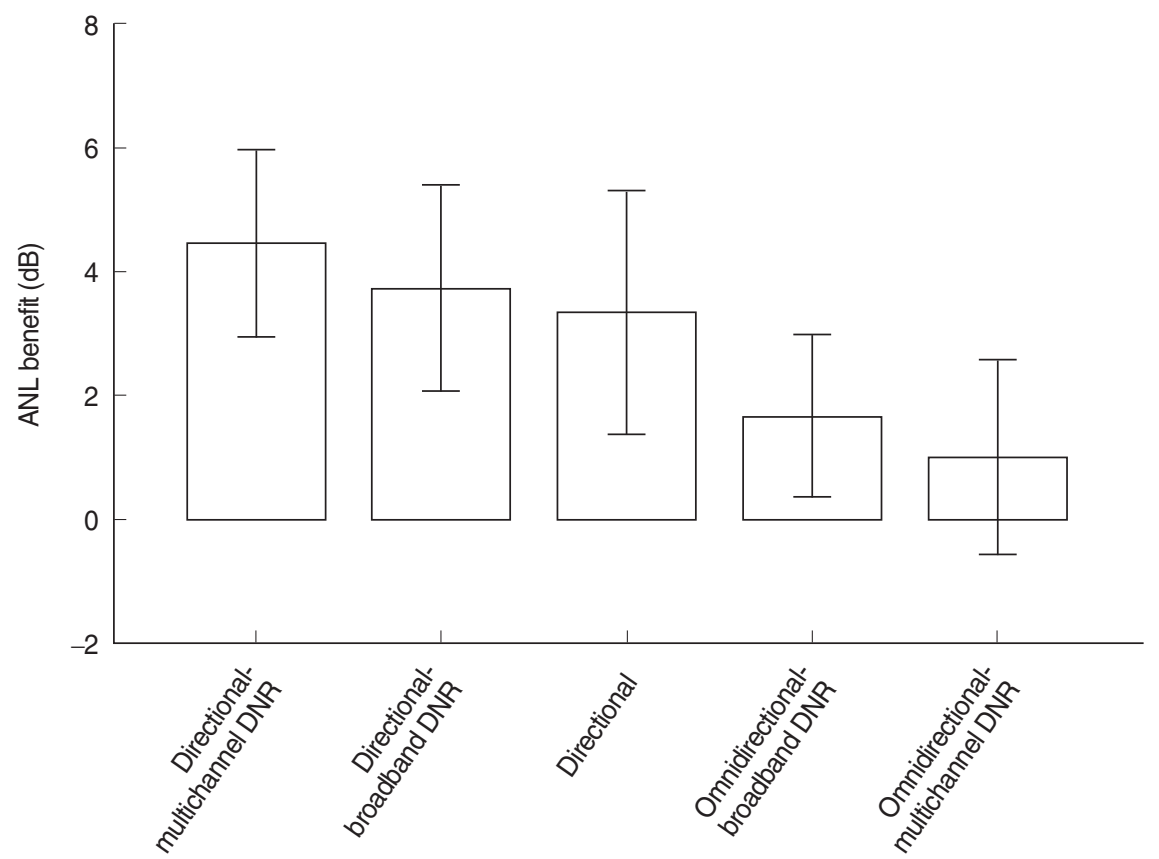

Fig. 5. The acceptable noise level (ANL) benefit under five different directionality/digital noise reduction (DNR) conditions for all participants. 
benefit to the DIR algorithm.

\section{DISCUSSION}

Taken together, our findings illustrate implementing the specific design of the present study, both DNR and DIR technologies yield significant improvements in the ANL measure. Consistent with previous studies $[8,19]$, the DNR and DIR benefit increased significantly from DNR to DIR to the DNR and DIR combination. Specifically, in ANL, compared with the omnidirectional-baseline condition, there is an average improvement of $3.3 \mathrm{~dB}$ with DIR, $1.33 \mathrm{~dB}$ with DNR, and $4.08 \mathrm{~dB}$ with both DIR and DNR conditions. These findings, although consistent with those of other studies [8,11,19-21], indicate the DNR provides comfort listening in noise for subjects. Although DIR algorithms provide more improvement compared with DNR algorithms, the extra improvement is added to the DIR benefit in the resultant DNR algorithm.

For additional confirmation of our results as well as for determining the gain reduction in the results of the hearing aid used in the current study, we measured the gain frequency response of the hearing aid in various conditions by the two main signals of the Digital Speech and the Composite Noise stimuli (as speech and noise stimuli, respectively) (Supplementary Table 1, Supplementary Figs. 1-5). The 2-cc coupler measurements showed the gain reduction at low frequencies for the omnidirectional-multichannel DNR condition when the hearing aid is presented by a Composite Noise stimulus. Although for the omnidirectionalbroadband DNR condition, there is a gain reduction over all frequency ranges. Thus, it is expected the ANL for the broadband DNR condition is less than the ANL for the omnidirectionalmultichannel DNR condition, a finding that was observed in the study. This conclusion is applicable only for the two omnidirectional DNR conditions rather than the two directional DNR conditions. Even though there is more gain reduction for the omnidirectional-broadband DNR condition when compared with the omnidirectional-multichannel DNR condition, there is no significant difference between ANLs for these conditions when the DIR algorithm is added to the DNR algorithm. It seems that, with the specific hearing aid used in this study, the DIR algorithm reduces noise more effectively than did the DNR algorithms. Therefore, it appears that although the DNR algorithm provides noise reduction and listening comfort in noise, the DIR algorithm for patients is more effective than is the DNR algorithm. This result may be associated with the different procedure of DSP activation in the hearing aid. In modern hearing aids, there are different interactions among various DSP algorithms (i.e., amplitude compression, DNR, and DIR) when the hearing aid is exposed to the various signals of speech and noise [22]. In the present study, there is more ANL benefit for the DIR algorithm compared with the DNR in both the separation and the combination modes. In the DIR condition, the average participant receives both lower noise and clear speech. The proportional contribution of the DIR algorithm in the ANL benefit is considerably more than the DNR algorithm (3.33 dB vs. 1.33 $\mathrm{dB})$, so that this additional ANL benefit is not provided when the DNR algorithm is added to the DIR algorithm (4.08 dB), a finding also reported by similar studies $[8,19]$. This result suggests the ANL can demonstrate the net effect of various DSP interactions inside hearing aids in clinical practice.

The directional-multichannel DNR condition is the most effective hearing aid condition for average participants regarding comfort. However, the omnidirectional-baseline condition is the worst condition. It has been shown DNR algorithms provide listening comfort in noise [5]. Also, our data is consistent with similar studies conducted by Mueller et al. [11] and Freyaldenhoven et al. [23], which showed an average ANL improvement of $3.5 \mathrm{~dB}$ and $4.2 \mathrm{~dB}$, respectively. The ANL improvement in our study is 4.44 $\mathrm{dB}$ and $3.72 \mathrm{~dB}$ for the directional-multichannel and the broadband DNR conditions, respectively. The results of the present study are consistent with those of other studies $[8,11,19,21,24]$ and suggest it is most appropriate to use of both DIR and DNR algorithms. However, these results are limited to our specific study design as well as the laboratory findings because it has been shown the subject benefit depends on both the type of DSP employed by the hearing aid $[8,11]$ and there may not DSP benefits in real life conditions because of reverberation or different types of noises. Certainly, there were differences in the measurements of directional benefits obtained under laboratory and real life conditions. Although the present study showed the benefits of listening comfort from the DNR and DIR, this finding is limited to both the specific hearing aid fitted for all study participants and the specific laboratory procedure applied in this study. In the present study, a condition was evaluated in which the speech signal and noise were present from the front and back, respectively. In the real life condition, there are many noise sources from different directions in which a subject with hearing aid experience most challenging listening condition. On the other hand, the closed fitting was performed in the present study since the open fitting has a deteriorating effect on the DNR and DIR benefit [14-16]. Also, there are various strategies for activating the DNR or the DIR in various hearing aids. In addition, the gain reduction of the DNR system is different among the variety of hearing aids on the market. Thus, in real life conditions since there are various sound sources with different locations and reverberation, there would be different results. These observations prove the important role of the ANL for evaluating the benefit from DSP algorithms of various hearing aids.

The DSP algorithms, DIR and DNR, provide listening comfort, especially for people with hearing aid experience whereas these algorithms do not deteriorate speech intelligibility in noise. The benefit from DNR and DIR algorithms depends on how DSP settings are adjusted. 


\section{CONFLICT OF INTEREST}

No potential conflict of interest relevant to this article was reported.

\section{SUPPLEMENTARY MATERIALS}

Supplementary materials can be found via https://doi.org/10. 21053/ceo.2018.00052.

\section{REFERENCES}

1. Kochkin S. MarkeTrak VI: consumers rate improvements sought in hearing instruments. Hear Rev. 2002 Nov;9(11):18-22.

2. McCormack A, Fortnum $H$. Why do people fitted with hearing aids not wear them? Int J Audiol. 2013 May;52(5):360-8.

3. Ng EH, Rudner M, Lunner T, Pedersen MS, Ronnberg J. Effects of noise and working memory capacity on memory processing of speech for hearing-aid users. Int J Audiol. 2013 Jul;52(7):433-41.

4. Obleser J, Wostmann M, Hellbernd N, Wilsch A, Maess B. Adverse listening conditions and memory load drive a common alpha oscillatory network. J Neurosci. 2012 Sep;32(36):12376-83.

5. Ricketts TA, Hornsby BW. Sound quality measures for speech in noise through a commercial hearing aid implementing digital noise reduction. JAm Acad Audiol. 2005 May;16(5):270-7.

6.Alcantara JL, Moore BC, KuhnelV, Launer S. Evaluation of the noise reduction system in a commercial digital hearing aid. Int $\mathrm{J}$ Audiol. 2003 Jan;42(1):34-42.

7. Boymans M, Dreschler WA. Field trials using a digital hearing aid with active noise reduction and dual-microphone directionality. Audiology. 2000 Sep-Oct;39(5):260-8.

8. Peeters H, Kuk F, Lau CC, Keenan D. Subjective and objective evaluation of noise management algorithms. J Am Acad Audiol. 2009 Feb;20(2):89-98.

9. Dillon H. Hearing aids. 2 ed. Sydney: Boomerang Press; 2012.

10. Nabelek AK, Tucker FM, Letowski TR. Toleration of background noises: relationship with patterns of hearing aid use by elderly persons. J Speech Hear Res. 1991 Jun;34(3):679-85.

11. Mueller HG, Weber J, Hornsby BW. The effects of digital noise re- duction on the acceptance of background noise.Trends Amplif. 2006 Jun;10(2):83-93.

12. Neher T, Grimm G, Hohmann V. Perceptual consequences of different signal changes due to binaural noise reduction: do hearing loss and working memory capacity play a role? Ear Hear. 2014 Sep-Oct; 35(5):e213-27.

13. Ansari NN, Naghdi S, Hasson S, Valizadeh L, Jalaie S. Validation of a Mini-Mental State Examination (MMSE) for the Persian population: a pilot study. Appl Neuropsychol. $2010 \mathrm{Jul} ; 17(3): 190-5$.

14. Kuk F, Keenan D. How do vents affect hearing aid performance? Hear Rev. 2006 Feb;13(2):34-42.

15. Magnusson L, Claesson A, Persson M,Tengstrand T. Speech recognition in noise using bilateral open-fit hearing aids: the limited benefit of directional microphones and noise reduction. Int J Audiol. 2013 Jan;52(1):29-36.

16. Winkler A, Latzel M, Holube I. Open versus closed hearing-aid fittings: a literature review of both fitting approaches. Trends Hear. 2016 Feb;20:2331216516631741.

17. Kuk F, Peeters H, Lau C, Korhonen P. Effect of maximum power output and noise reduction on speech recognition in noise. J Am Acad Audiol. 2011 May;22(5):265-73.

18. Ahmadi A, Fatahi J, Keshani A, Jalilvand H, Modarresi Y, Jalaie S. Developing and evaluating the reliability of acceptable noise level test in Persian language. Sci J Rehabil Med. 2015;4(2):109-17.

19. Lowery KJ, Plyler PN.The effects of noise reduction technologies on the acceptance of background noise. J Am Acad Audiol. 2013 Sep;24(8):649-59.

20. Desjardins JL. The effects of hearing aid directional microphone and noise reduction processing on listening effort in older adults with hearing loss. J Am Acad Audiol. 2016 Jan;27(1):29-41.

21. Nordrum S, Erler S, Garstecki D, Dhar S. Comparison of performance on the hearing in noise test using directional microphones and digital noise reduction algorithms. Am J Audiol. 2006 Jun;15(1):81-91.

22. Brons I, Houben R, Dreschler WA. Acoustical and perceptual comparison of noise reduction and compression in hearing aids. J Speech Lang Hear Res. 2015 Aug;58(4):1363-76.

23. Freyaldenhoven MC, Nabelek AK, Burchfield SB, Thelin JW. Acceptable noise level as a measure of directional hearing aid benefit. J Am Acad Audiol. 2005 Apr;16(4):228-36.

24. Aghsoleimani M, Jalilvand H, Mahdavi ME, Nazeri AR, Kamali M. The acceptable noise level benefit from directionality for listeners with severe hearing loss. Clin Exp Otorhinolaryngol. 2018 Sep;11(3): 166-73. 